\title{
Exact Explicit Solutions and Conservation Laws for a Coupled Zakharov-Kuznetsov System
}

\author{
Chaudry Masood Khalique \\ Department of Mathematical Sciences, International Institute for Symmetry Analysis and Mathematical Modelling, \\ North-West University, Mafikeng Campus, Private Bag X 2046, Mmabatho 2735, South Africa
}

Correspondence should be addressed to Chaudry Masood Khalique; masood.khalique@nwu.ac.za

Received 11 May 2013; Accepted 17 June 2013

Academic Editor: Mufid Abudiab

Copyright (c) 2013 Chaudry Masood Khalique. This is an open access article distributed under the Creative Commons Attribution License, which permits unrestricted use, distribution, and reproduction in any medium, provided the original work is properly cited.

We study a coupled Zakharov-Kuznetsov system, which is an extension of a coupled Korteweg-de Vries system in the sense of the Zakharov-Kuznetsov equation. Firstly, we obtain some exact solutions of the coupled Zakharov-Kuznetsov system using the simplest equation method. Secondly, the conservation laws for the coupled Zakharov-Kuznetsov system will be constructed by using the multiplier approach.

\section{Introduction}

It is well known that the two-dimensional generalizations of the Korteweg-de Vries (KdV) equation

$$
u_{t}+a u u_{x}+u_{x x x}=0
$$

are the Kadomtsev-Petviashivili (KP) equation and the Zakharov-Kuznetsov (ZK) equation. The ZK equation

$$
u_{t}+a u u_{x}+b\left(u_{x x}+u_{y y}\right)_{x}=0
$$

governs the behaviour of weakly nonlinear ion-acoustic waves in a plasma comprising cold ions and hot isothermal electrons in the presence of a uniform magnetic field [1]. In [2] a new hierarchy of nonlinear evolution equations was derived, and one particular system of equations

$$
\begin{gathered}
u_{t}=\beta u_{x x x}+\alpha(u v)_{x}+\gamma(v w)_{x}, \\
v_{t}=\beta v_{x x x}+\lambda(w u)_{x}, \\
w_{t}=\beta w_{x x x}+\lambda(u v)_{x},
\end{gathered}
$$

where $\alpha, \beta, \gamma$, and $\lambda$ are constants, was later studied by [3]. This coupled KdV system (3a), (3b), and (3c) was extended to the new coupled ZK system

$$
\begin{gathered}
u_{t}-\alpha(u v)_{x}-\gamma(v w)_{x}-\beta\left(u_{x x}+u_{y y}\right)_{x}=0, \\
v_{t}-\lambda(w u)_{x}-\beta\left(v_{x x}+v_{y y}\right)_{x}=0, \\
w_{t}-\lambda(u v)_{x}-\beta\left(w_{x x}+w_{y y}\right)_{x}=0,
\end{gathered}
$$

in the sense of the ZK Equation (2) in [1], and travelling wave solutions were determined using the extended tanhcoth method and sech method.

In the last few decades, several powerful methods have been introduced in the literature, which can be used to find exact solutions of nonlinear differential equations arising from physical problems. These methods include the inverse scattering transform method [4], the Darboux transformation [5], the Hirota's bilinear method [6], the Jacobi elliptic function expansion method $[7,8]$, the multiple-exp method [9], the sine-cosine method [10], the Lie symmetry method $[11,12]$, and the $\left(G^{\prime} / G\right)$-expansion method [13].

The purpose of this paper is to employ the simplest equation method $[14,15]$ to obtain some exact explicit solutions of the coupled Zakharov-Kuznetsov system (4a), 
(4b), and (4c). Furthermore, we derive conservation laws for (4a), (4b), and (4c) using the multiplier approach [16-18].

\section{Exact Solutions Using Simplest Equation Method}

In this section we employ the simplest equation method [14, 15] and obtain some exact explicit solutions of (4a), (4b), and (4c). The simplest equations that will be used in this paper are the Bernoulli and Riccati equations. It is well known that their solutions can be written in elementary functions. See, for example, [19].

By using the transformation

$$
z=k_{1} t+k_{2} x+k_{3} y+k_{4}
$$

where $k_{i}, i=1, \ldots, 4$, are constants, the coupled ZakharovKuznetsov system (4a), (4b), and (4c) transforms to a thirdorder coupled system of nonlinear ordinary differential equations (ODEs)

$$
\begin{aligned}
& \beta k_{2}^{3} E^{\prime \prime \prime}(z)+\beta k_{3}^{2} k_{2} E^{\prime \prime \prime}(z)+\alpha k_{2} F(z) E^{\prime}(z) \\
& -k_{1} E^{\prime}(z)+\alpha k_{2} E(z) F^{\prime}(z)+\gamma k_{2} G(z) F^{\prime}(z) \\
& +\gamma k_{2} F(z) G^{\prime}(z)=0 \\
& \beta k_{2}^{3} F^{\prime \prime \prime}(z)+\beta k_{3}^{2} k_{2} F^{\prime \prime \prime}(z)+\lambda k_{2} G(z) E^{\prime}(z) \\
& +\lambda k_{2} E(z) G^{\prime}(z)-k_{1} F^{\prime}(z)=0 \\
& \beta k_{2}^{3} G^{\prime \prime \prime}(z)+\beta k_{3}^{2} k_{2} G^{\prime \prime \prime}(z)+\lambda k_{2} F(z) E^{\prime}(z) \\
& +\lambda k_{2} E(z) F^{\prime}(z)-k_{1} G^{\prime}(z)=0 .
\end{aligned}
$$

We now present the simplest equation method for a system of three ODEs. Consider the solutions of (6a), (6b), and (6c) in the form

$$
\begin{aligned}
& E(z)=\sum_{i=0}^{M} A_{i}(H(z))^{i}, \\
& F(z)=\sum_{i=0}^{M} B_{i}(H(z))^{i}, \\
& G(z)=\sum_{i=0}^{M} C_{i}(H(z))^{i},
\end{aligned}
$$

where $H(z)$ satisfies the Bernoulli or Riccati equation, $M$ is a positive integer that can be determined by balancing procedure [15], and $A_{i}, B_{i}$, and $C_{i}(i=0,1, \ldots, M)$ are parameters to be determined.

The Bernoulli equation we consider in this paper is

$$
H^{\prime}(z)=a H(z)+b H^{2}(z),
$$

where $a$ and $b$ are constants. Its solution can be written as

$$
H(z)=a\left\{\frac{\cosh [a(z+C)]+\sinh [a(z+C)]}{1-b \cosh [a(z+C)]-b \sinh [a(z+C)]}\right\} .
$$

For the Riccati equation

$$
H^{\prime}(z)=a H^{2}(z)+b H(z)+c,
$$

where $a, b$, and $c$ are constants, we will use the solutions

$$
\begin{aligned}
H(z)= & -\frac{b}{2 a}-\frac{\theta}{2 a} \tanh \left[\frac{1}{2} \theta(z+C)\right], \\
H(z)= & -\frac{b}{2 a}-\frac{\theta}{2 a} \tanh \left(\frac{1}{2} \theta z\right) \\
& +\frac{\operatorname{sech}(\theta z / 2)}{C \cosh (\theta z / 2)-(2 a / \theta) \sinh (\theta z / 2)},
\end{aligned}
$$

where $\theta^{2}=b^{2}-4 a c$.

2.1. Solutions of (4a), (4b), and (4c) Using the Bernoulli Equation as the Simplest Equation. The balancing procedure yields $M=2$. Thus, the solutions of (6a), (6b), and (6c) are of the form

$$
\begin{gathered}
E(z)=A_{0}+A_{1} H+A_{2} H^{2}, \\
F(z)=B_{0}+B_{1} H+B_{2} H^{2}, \\
G(z)=C_{0}+C_{1} H+C_{2} H^{2} .
\end{gathered}
$$

Substituting (12a), (12b), and (12c) into (6a), (6b), and (6c) and making use of the Bernoulli equation (8) and then equating the coefficients of the functions $H^{i}$ to zero, we obtain an algebraic system of equations in terms of $A_{i}, B_{i}$, and $C_{i}(i=$ $0,1,2)$. Solving this system of algebraic equations, with the aid of Mathematica, one possible set of values of $A_{i}, B_{i}$, and $C_{i}(i=0,1,2)$ is

$$
\begin{gathered}
a=1, \quad b=3, \\
\alpha=-\frac{\gamma B_{2}{ }^{2}+54 \beta k_{2}{ }^{2} A_{2}+54 \beta k_{3}{ }^{2} A_{2}}{A_{2} B_{2}}, \\
\lambda=-\frac{54 \beta\left(k_{2}{ }^{2}+k_{3}{ }^{2}\right)}{A_{2}}, \\
A_{0}=\frac{A_{2} B_{0}}{B_{2}}, \quad A_{1}=\left(\frac{1}{3}\right) A_{2}, \quad B_{1}=\left(\frac{1}{3}\right) B_{2}, \\
k_{1}=-\frac{C_{0}=B_{0}, \quad C_{1}=\left(\frac{1}{3}\right) B_{2}, \quad B_{2},}{k_{2}\left(108 B_{0} k_{3}{ }^{2}-k_{2}{ }^{2} B_{2}-k_{3}{ }^{2} B_{2}+108 B_{0} k_{2}{ }^{2}\right)}
\end{gathered} .
$$


As a result, a solution of (4a), (4b), and (4c) is

$$
\begin{aligned}
& u(t, x, y) \\
& =A_{0}+A_{1} a\left\{\frac{\cosh [a(z+C)]+\sinh [a(z+C)]}{1-b \cosh [a(z+C)]-b \sinh [a(z+C)]}\right\} \\
& \quad+A_{2} a^{2}\left\{\frac{\cosh [a(z+C)]+\sinh [a(z+C)]}{1-b \cosh [a(z+C)]-b \sinh [a(z+C)]}\right\}^{2},
\end{aligned}
$$

$$
\begin{aligned}
v(t, x, y) & \\
= & B_{0}+B_{1} a\left\{\frac{\cosh [a(z+C)]+\sinh [a(z+C)]}{1-b \cosh [a(z+C)]-b \sinh [a(z+C)]}\right\} \\
& +B_{2} a^{2}\left\{\frac{\cosh [a(z+C)]+\sinh [a(z+C)]}{1-b \cosh [a(z+C)]-b \sinh [a(z+C)]}\right\}^{2},
\end{aligned}
$$

$$
\begin{aligned}
& w(t, x, y) \\
& =C_{0}+C_{1} a\left\{\frac{\cosh [a(z+C)]+\sinh [a(z+C)]}{1-b \cosh [a(z+C)]-b \sinh [a(z+C)]}\right\} \\
& \quad+C_{2} a^{2}\left\{\frac{\cosh [a(z+C)]+\sinh [a(z+C)]}{1-b \cosh [a(z+C)]-b \sinh [a(z+C)]}\right\}^{2},
\end{aligned}
$$

where $z=k_{1} t+k_{2} x+k_{3} y+k_{4}$.

2.2. Solutions of (4a), (4b), and (4c) Using Riccati Equation as the Simplest Equation. The balancing procedure yields $M=2$ and so the solutions of (6a), (6b), and (6c) are of the form

$$
\begin{aligned}
& E(z)=A_{0}+A_{1} H+A_{2} H^{2}, \\
& F(z)=B_{0}+B_{1} H+B_{2} H^{2}, \\
& G(z)=C_{0}+C_{1} H+C_{2} H^{2} .
\end{aligned}
$$

Substituting (15a), (15b), and (15c) into (6a), (6b), and (6c) and using (10), we obtain an algebraic system of equations in terms of $A_{i}, B_{i}$, and $C_{i}(i=0,1,2)$ by equating all coefficients of the functions $H^{i}$ to zero. Solving the resultant system, one possible set of values is

$$
\begin{gathered}
a=1, \quad b=3, \quad c=1, \\
\alpha=\frac{\gamma B_{2}{ }^{2}-6 \beta k_{2}^{2} A_{2}-6 \beta k_{3}{ }^{2} A_{2}}{B_{2} A_{2}}, \\
\lambda=\frac{6 \beta\left(k_{2}{ }^{2}+k_{3}{ }^{2}\right)}{A_{2}}, \\
A_{0}=\frac{A_{2} B_{0}}{B_{2}}, \quad A_{1}=3 A_{2}, \quad B_{1}=3 B_{2},
\end{gathered}
$$

$$
\begin{gathered}
C_{0}=-B_{0}, \quad C_{1}=-3 B_{2}, \quad C_{2}=-B_{2}, \\
k_{1}=-\frac{\beta k_{2}\left(-17 k_{3}{ }^{2} B_{2}-17 k_{2}{ }^{2} B_{2}+12 B_{0} k_{3}{ }^{2}+12 B_{0} k_{2}{ }^{2}\right)}{B_{2}} .
\end{gathered}
$$

Consequently, the solutions of (4a), (4b), and (4c) are

$$
\begin{aligned}
u(t, x, y)= & A_{0}+A_{1}\left\{-\frac{b}{2 a}-\frac{\theta}{2 a} \tanh \left[\frac{1}{2} \theta(z+C)\right]\right\} \\
& +A_{2}\left\{-\frac{b}{2 a}-\frac{\theta}{2 a} \tanh \left[\frac{1}{2} \theta(z+C)\right]\right\}^{2}, \\
v(t, x, y)= & B_{0}+B_{1}\left\{-\frac{b}{2 a}-\frac{\theta}{2 a} \tanh \left[\frac{1}{2} \theta(z+C)\right]\right\} \\
& +B_{2}\left\{-\frac{b}{2 a}-\frac{\theta}{2 a} \tanh \left[\frac{1}{2} \theta(z+C)\right]\right\}^{2}, \\
w(t, x, y)= & C_{0}+C_{1}\left\{-\frac{b}{2 a}-\frac{\theta}{2 a} \tanh \left[\frac{1}{2} \theta(z+C)\right]\right\} \\
& +C_{2}\left\{-\frac{b}{2 a}-\frac{\theta}{2 a} \tanh \left[\frac{1}{2} \theta(z+C)\right]\right\}^{2},
\end{aligned}
$$

$u(t, x, y)$

$$
=A_{0}+A_{1}\left\{-\frac{b}{2 a}-\frac{\theta}{2 a} \tanh \left(\frac{1}{2} \theta z\right)\right.
$$$$
\left.+\frac{\operatorname{sech}(\theta z / 2)}{C \cosh (\theta z / 2)-(2 a / \theta) \sinh (\theta z / 2)}\right\}
$$$$
+A_{2}\left\{-\frac{b}{2 a}-\frac{\theta}{2 a} \tanh \left(\frac{1}{2} \theta z\right)\right.
$$

$$
\left.+\frac{\operatorname{sech}(\theta z / 2)}{C \cosh (\theta z / 2)-(2 a / \theta) \sinh (\theta z / 2)}\right\}^{2},
$$

$$
v(t, x, y)=B_{0}+B_{1}\left\{-\frac{b}{2 a}-\frac{\theta}{2 a} \tanh \left(\frac{1}{2} \theta z\right)\right.
$$

$$
\left.+\frac{\operatorname{sech}(\theta z / 2)}{C \cosh (\theta z / 2)-(2 a / \theta) \sinh (\theta z / 2)}\right\}
$$$$
+B_{2}\left\{-\frac{b}{2 a}-\frac{\theta}{2 a} \tanh \left(\frac{1}{2} \theta z\right)\right.
$$$$
\left.+\frac{\operatorname{sech}(\theta z / 2)}{C \cosh (\theta z / 2)-(2 a / \theta) \sinh (\theta z / 2)}\right\}^{2},
$$ 


$$
\begin{aligned}
w(t, x, y) & \\
=C_{0}+C_{1}\left\{-\frac{b}{2 a}-\frac{\theta}{2 a} \tanh \left(\frac{1}{2} \theta z\right)\right. & \\
& \left.+\frac{\operatorname{sech}(\theta z / 2)}{C \cosh (\theta z / 2)-(2 a / \theta) \sinh (\theta z / 2)}\right\} \\
+C_{2}\{- & \frac{b}{2 a}-\frac{\theta}{2 a} \tanh \left(\frac{1}{2} \theta z\right) \\
& \left.+\frac{\operatorname{sech}(\theta z / 2)}{C \cosh (\theta z / 2)-(2 a / \theta) \sinh (\theta z / 2)}\right\}^{2},
\end{aligned}
$$

where $z=k_{1} t+k_{2} x+k_{3} y+k_{4}$.

\section{Conservation Laws of (4a), (4b), and (4c)}

In this section we derive conservation laws for the coupled Zakharov-Kuznetsov system (4a), (4b), and (4c). The multiplier approach will be used. For details the reader is referred to $[11,16-18]$.

In our case we obtain multipliers [17] of the form

$$
\Lambda_{1}=f_{1}(y), \quad \Lambda_{2}=f_{2}(y), \quad \Lambda_{3}=f_{3}(y),
$$

and corresponding to the above multipliers we then obtain the following conserved vectors [17] of (4a), (4b), and (4c):

$$
\begin{aligned}
& T_{1}^{t}= f_{1}(y) u, \\
& T_{2}^{x}= \frac{1}{3}\left\{-3 \alpha f_{1}(y) u v-\beta f_{1}^{\prime \prime}(y) u-3 \gamma f_{1}(y) v w\right. \\
&\left.\quad-3 \beta f_{1}(y) u_{x x}+\beta f_{1}^{\prime}(y) u_{y}-\beta f_{1}(y) u_{y y}\right\}, \\
& T_{3}^{y}= \frac{1}{3}\left\{\beta f_{1}^{\prime}(y) u_{x}-2 \beta f_{1}(y) u_{x y}\right\}, \\
& T_{2}^{t}= f_{2}(y) v, \\
& T_{2}^{x}= \frac{1}{3}\left\{-3 \lambda f_{2}(y) u w-\beta f_{2}^{\prime \prime}(y) v-3 \beta f_{2}(y) v_{x x}\right. \\
&\left.+\beta f_{2}^{\prime}(y) v_{y}-\beta f_{2}(y) v_{y y}\right\}, \\
& T_{2}^{y}= \frac{1}{3}\left\{\beta f_{2}^{\prime}(y) v_{x}-2 \beta f_{2}(y) v_{x y}\right\}, \\
& T_{3}^{t}= f_{3}(y) w, \\
& T_{3}^{x}= \frac{1}{3}\left\{-3 \lambda f_{3}(y) u v-\beta f_{3}^{\prime \prime}(y) w-3 \beta f_{3}(y) w_{x x}\right. \\
&\left.\quad+\beta f_{3}^{\prime}(y) w_{y}-\beta f_{3}(y) w_{y y}\right\}, \\
& T_{3}^{y}=\frac{1}{3}\left\{\beta f_{3}^{\prime}(y) w_{x}-2 \beta f_{3}(y) w_{x y}\right\} .
\end{aligned}
$$

It should be noted that due to the presence of the arbitrary function $f(y)$ in the multipliers there are infinitely many conservation laws for the coupled Zakharov-Kuznetsov system (4a), (4b), and (4c).

\section{Concluding Remarks}

In this paper we obtained some exact solutions of the coupled Zakharov-Kuznetsov system (4a), (4b), and (4c) by the aid of the simplest equation method. The solutions obtained are solitary waves. Moreover, the conservation laws for the coupled Zakharov-Kuznetsov system (4a), (4b), and (4c) were also derived by using the multiplier approach.

\section{References}

[1] M. Wei and S. Tang, "Exact explicit traveling wave solutions for a new coupled ZK system," The Journal of Applied Analysis and Computation, vol. 1, no. 2, pp. 267-277, 2011.

[2] Z. Qin, "A finite-dimensional integrable system related to a new coupled KdV hierarchy," Physics Letters A, vol. 355, no. 6, pp. 452-459, 2006.

[3] J. Wu, "New explicit traveling wave solutions for three nonlinear evolution equations," Applied Mathematics and Computation, vol. 217, no. 4, pp. 1764-1770, 2010.

[4] M. J. Ablowitz and P. A. Clarkson, Solitons, Nonlinear Evolution Equations and Inverse Scattering, vol. 149 of London Mathematical Society Lecture Note Series, Cambridge University Press, Cambridge, Mass, USA, 1991.

[5] V. B. Matveev and M. A. Salle, Darboux Transformations and Solitons, Springer Series in Nonlinear Dynamics, Springer, Berlin, Germany, 1991.

[6] R. Hirota, The Direct Method in Soliton Theory, vol. 155 of Cambridge Tracts in Mathematics, Cambridge University Press, Cambridge, Mass, USA, 2004.

[7] D. Lü, "Jacobi elliptic function solutions for two variant Boussinesq equations," Chaos, Solitons \& Fractals, vol. 24, no. 5, pp. 1373-1385, 2005.

[8] Z. Yan, "Abundant families of Jacobi elliptic function solutions of the (2+1)-dimensional integrable Davey-Stewartson-type equation via a new method," Chaos, Solitons \& Fractals, vol. 18, no. 2, pp. 299-309, 2003.

[9] W.-X. Ma, T. Huang, and Y. Zhang, "A multiple exp-function method for nonlinear differential equations and its application," Physica Scripta, vol. 82, no. 6, Article ID 065003, 2010.

[10] A.-M. Wazwaz, "The tanh and the sine-cosine methods for compact and noncompact solutions of the nonlinear KleinGordon equation," Applied Mathematics and Computation, vol. 167, no. 2, pp. 1179-1195, 2005.

[11] P. J. Olver, Applications of Lie Groups to Differential Equations, vol. 107 of Graduate Texts in Mathematics, Springer, New York, NY, USA, 2nd edition, 1993.

[12] K. R. Adem and C. M. Khalique, "Exact solutions and conservation laws of a (2+1)-dimensional nonlinear KP-BBM equation," Abstract and Applied Analysis, vol. 2013, Article ID 791863, 5 pages, 2013.

[13] M. Wang, X. Li, and J. Zhang, "The $\left(G^{\prime} / G\right)$-expansion method and travelling wave solutions of nonlinear evolution equations in mathematical physics," Physics Letters A, vol. 372, no. 4, pp. 417-423, 2008. 
[14] N. A. Kudryashov, "Simplest equation method to look for exact solutions of nonlinear differential equations," Chaos, Solitons \& Fractals, vol. 24, no. 5, pp. 1217-1231, 2005.

[15] N. K. Vitanov, "Application of simplest equations of Bernoulli and Riccati kind for obtaining exact traveling-wave solutions for a class of PDEs with polynomial nonlinearity," Communications in Nonlinear Science and Numerical Simulation, vol. 15, no. 8, pp. 2050-2060, 2010.

[16] H. Steudel, "Über die Zuordnung zwischen Invarianzeigenschaften und Erhaltungssätzen," Zeitschrift für Naturforschung, vol. 17, pp. 129-132, 1962.

[17] S. C. Anco and G. Bluman, "Direct construction method for conservation laws of partial differential equations. I. Examples of conservation law classifications," European Journal of Applied Mathematics, vol. 13, no. 5, pp. 545-566, 2002.

[18] M. Anthonyrajah and D. P. Mason, "Conservation laws and invariant solutions in the Fanno model for turbulent compressible flow," Mathematical \& Computational Applications, vol. 15, no. 4, pp. 529-542, 2010.

[19] A. R. Adem and C. M. Khalique, "Symmetry reductions, exact solutions and conservation laws of a new coupled KdV system," Communications in Nonlinear Science and Numerical Simulation, vol. 17, no. 9, pp. 3465-3475, 2012. 


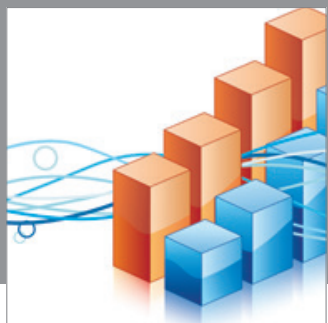

Advances in

Operations Research

mansans

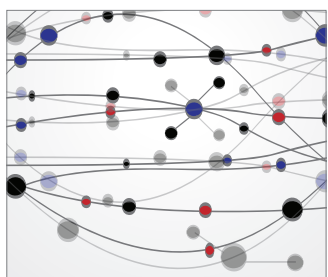

The Scientific World Journal
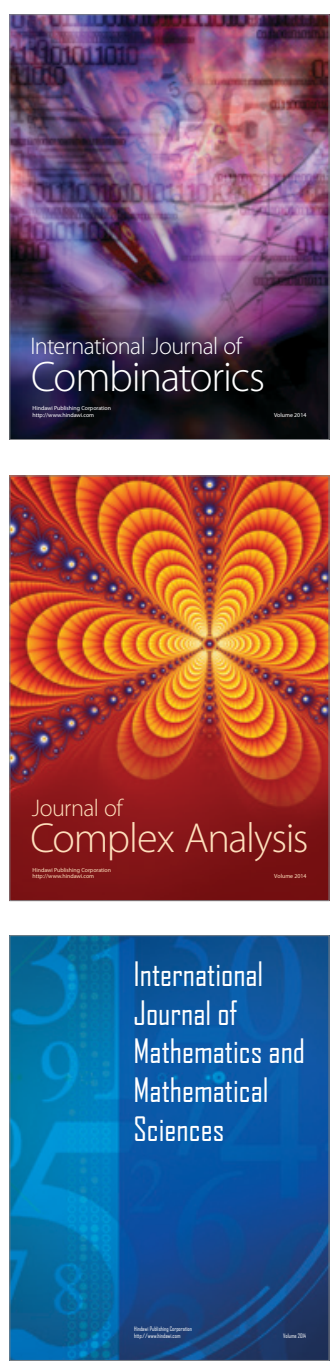
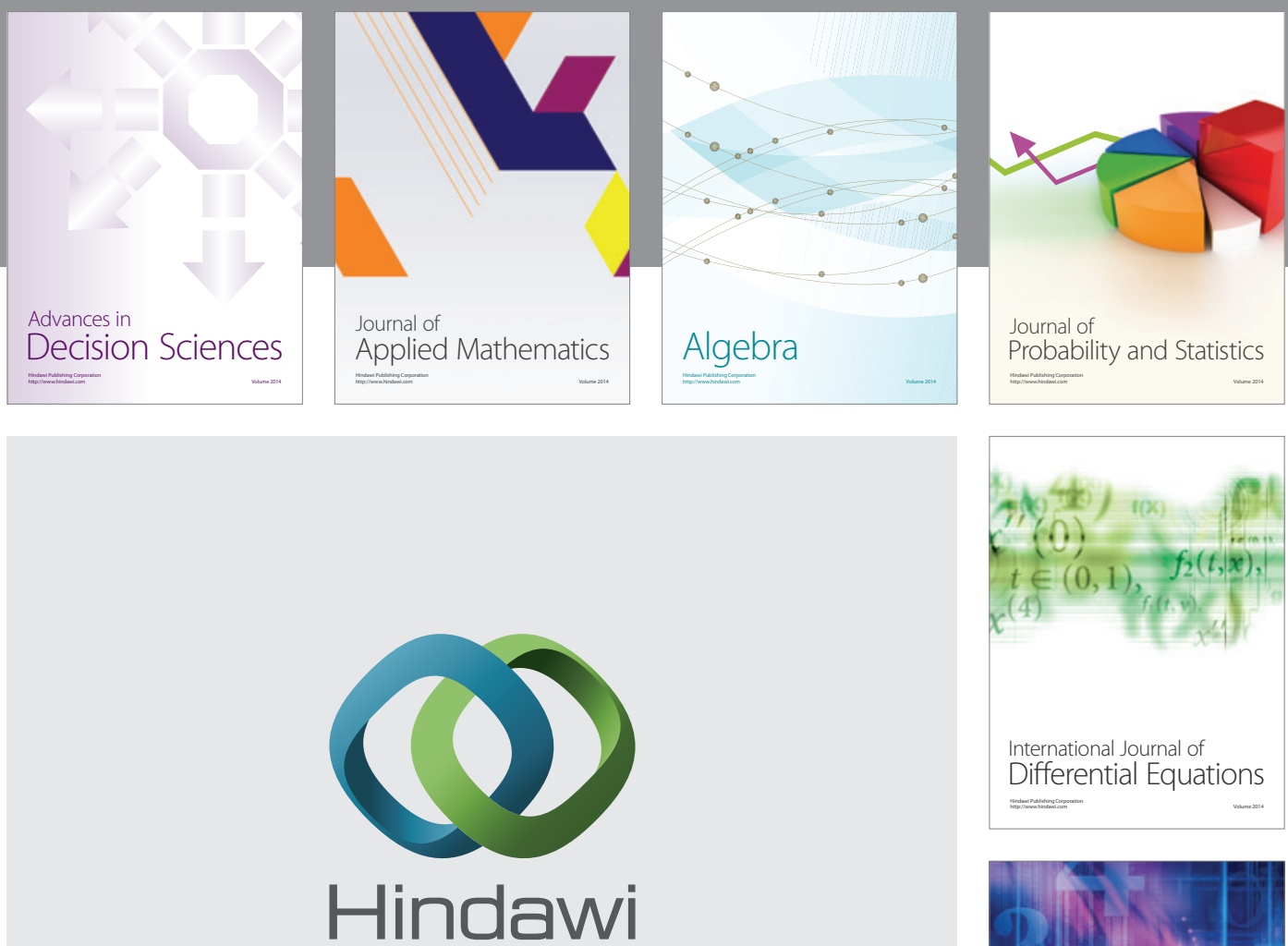

Submit your manuscripts at http://www.hindawi.com
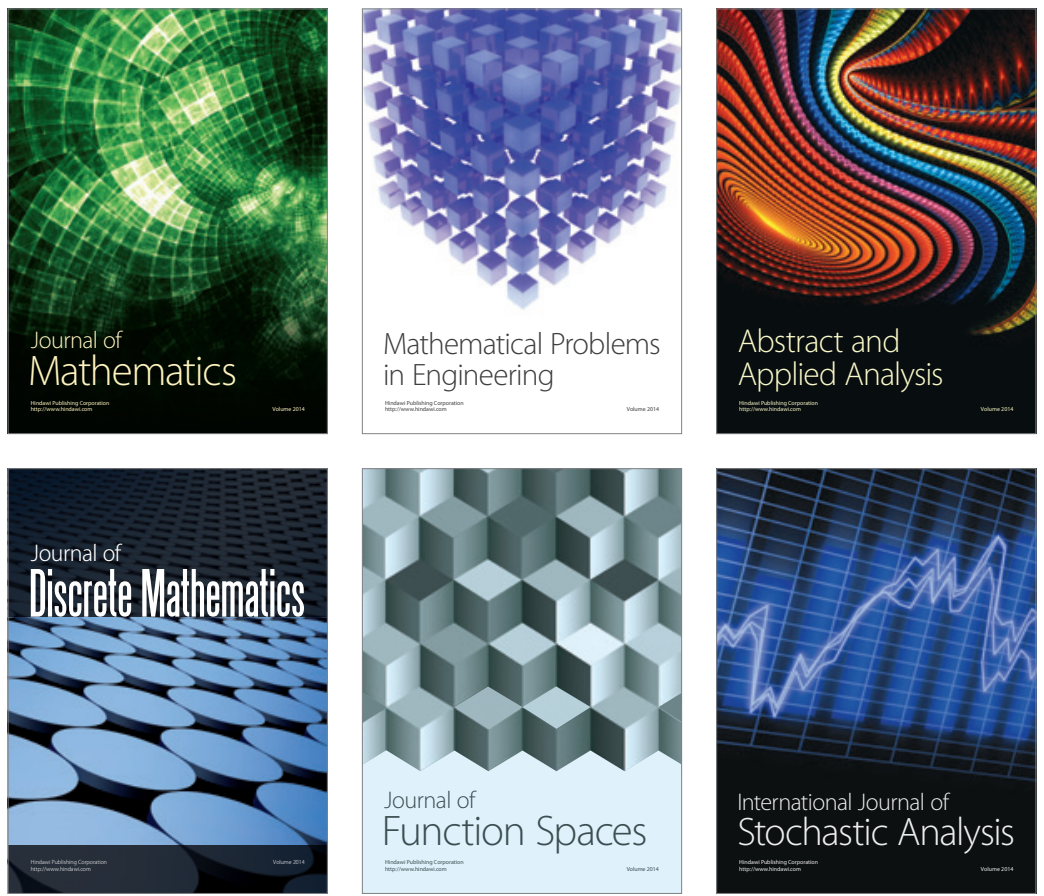

Journal of

Function Spaces

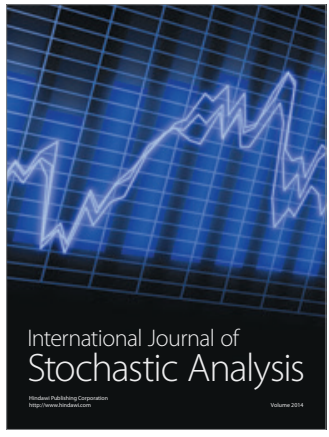

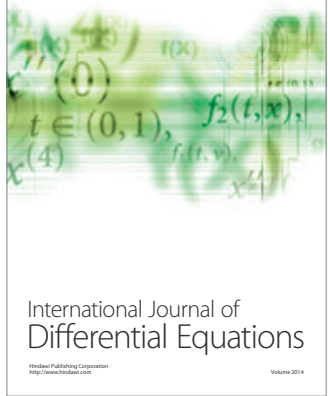
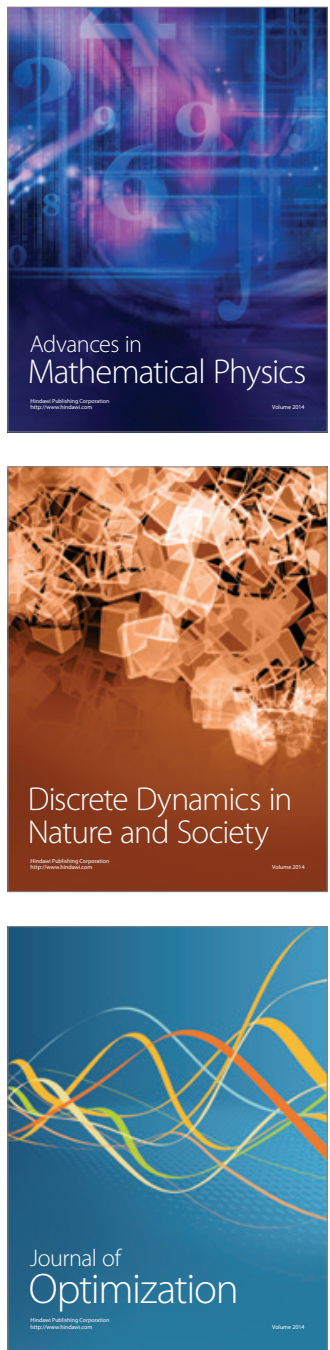